\title{
Assessment of knowledge about biobanking among healthcare students and their willingness to donate biospecimens
}

Leena Merdad', Lama Aldakhil2 ${ }^{2}$ Rawan Gadi ${ }^{1}$, Mourad Assidi ${ }^{3,4}$, Salina Y. Saddick ${ }^{5}$, Adel Abuzenadah ${ }^{3,4}$, Jim Vaught ${ }^{6,7}$, Abdelbaset Buhmeida $^{3^{*}}$ and Mohammed H. Al-Qahtani ${ }^{3}$

\begin{abstract}
Background: Biobanks and biospecimen collections are becoming a primary means of delivering personalized diagnostics and tailoring individualized therapeutics. This shift towards precision medicine (PM) requires interactions among a variety of stakeholders, including the public, patients, healthcare providers, government, and donors. Very few studies have investigated the role of healthcare students in biobanking and biospecimen donations. The main aims of this study were (1) to evaluate the knowledge of senior healthcare students about biobanks and (2) to assess the students' willingness to donate biospecimens and the factors influencing their attitudes.
\end{abstract}

Methods: A cross-sectional study was conducted among senior healthcare students at King Abdulaziz University (KAU), Saudi Arabia. The data were obtained using a self-administered questionnaire in English. In addition to the respondents' biographical data section, the questionnaire assessed the respondents' general knowledge about biobanking, the factors influencing their willingness to donate biospecimens to biobanks and their general attitudes towards biomedical research.

Results: A total of 597 senior healthcare students were included in the study. The general knowledge score was $3.2( \pm 1.6)$ out of 7 . Only approximately $44 \%$ and $27 \%$ of students were aware of the terms "Human Genome Project" (HGP) and "biobank," respectively. The majority of the students (89\%) were willing to donate biospecimens to biobanks. Multiple factors were significantly associated with their willingness to donate, including their perceived general health $(p<0.001)$, past experience with both tissue testing $(p<0.04)$ and tissue donation $(p<0.001)$, biobanking knowledge score $(p<0.001)$ and biomedical research attitude score $(p<0.001)$. The main reasons for students' willingness to donate were advancement of medical research and societal benefits, whereas misuse of biospecimens and confidentiality breaches were the main reasons for a reluctance to donate.

Conclusion: Despite their strong willingness to donate biospecimens, students exhibited a notable lack of knowledge about biobanking and the HGP. To expedite the transition towards PM, it is highly recommended to enhance healthcare curricula by including more educational and awareness programmes to familiarize students with OMICs technologies in addition to the scope of research and clinical applications.

Keywords: Biobank, Survey, Willingness to donate, Knowledge, Biospecimens, Medical students, Precision medicine

\footnotetext{
* Correspondence: abuhme@utu.fi

${ }^{3}$ Center of Excellence in Genomic Medicine Research, King Abdulaziz

University, P.O. Box 80216, Jeddah 21589, Saudi Arabia

Full list of author information is available at the end of the article
} 


\section{Background}

One of the major outcomes of the Human Genome Project (HGP) is the advancement of biobanks, which are collections of biospecimens from patients and volunteer donors combined with their personal information, clinicopathological features and even research data $[1,2]$. In the post-genomic era, biospecimen collection is becoming the main mechanism of delivering personalized diagnostics and tailored individualized therapeutics [3, 4]. Therefore, biobanks are considered the primary resource that will help shape the future of human health through precision medicine (PM). Briefly, PM can be defined as the right treatment for the right patient at the right time, every time. To be implemented efficiently, PM relies mainly on elucidating the dynamic interplay between the environment (living, non-living), lifestyle and social well-being and an individual's genomic make up in both health and disease status. Therefore, improving our understanding of the environment and lifestyle components, their digitalization, and especially assessing their contribution either a prevention or risk factor is crucial to developing customized disease-specific and/or public health strategies. Therefore, implementing standard operating procedures (SOPs) for sampling, collecting and processing biospecimens as well as considering the related bioethics and principles of responsible data sharing are thus paramount to attaining effective prevention and PM.

The current shift towards precision medicine (PM) requires comprehensive interactions between a variety of stakeholders involved in biobanking, including the public, patients, healthcare providers, the government, and donors $[5,6]$. Support, understanding and collaboration between these stakeholders are key to the survival and proper functioning of biobanks. Several studies have described the role of the public $[7,8]$ and patients [9-11] in biobanking and tissue donation, but very few studies have investigated the role of healthcare professionals.

Public support, understanding and active involvement are crucial to the survival of biobanks and genetic research in general. Therefore, a suitable assessment of the public's knowledge and attitudes can provide researchers with information about how to approach potential donors and seek their approval [7]. In addition, depending on whether the samples received are obtained from living or deceased donors, the collection of biospecimens requires consent from the donor or the donor's relatives, respectively. A study conducted in Sweden that assessed the willingness of the general public to donate tissue samples concluded that $78 \%$ agreed to donate and store biospecimens for subsequent research use [7]. Researchers from Italy indicated that $86 \%$ of individuals approved of sample donation for research purposes [8].

Despite these positive attitudes towards sample donation, there is still limited understanding of the definition, role, importance, scale, and governance of biobanks and their contributions to medical research, both globally and regionally [12-15]. Several factors have been demonstrated to affect willingness to participate in biomedical research and/or donate biospecimens, including age $[14,16-19]$, education $[1,7,8,20]$, and concerns about lack of confidentiality [21]. In addition, religious beliefs and cultural trends have been reported to be influential factors [22, 23].

Ensuring continuous development and innovation in PM and biomedical research requires increased biobanking knowledge, training and continuous education of healthcare professionals, including undergraduate and graduate students, who are the future leaders of the field $[24,25]$. A better understanding of healthcare students' baseline knowledge of biobanks and their willingness to donate biospecimens is therefore essential. Very few studies have assessed knowledge about biobanks and willingness to donate biospecimens among healthcare workers, both in general and in Saudi Arabia in particular. The main objective of the current study was to assess the knowledge of senior healthcare students about biobanks and their willingness to donate biospecimens, with a focus on the main factors that might influence their knowledge, attitudes and practices in the PM era. This study specifically targets senior students at King Abdulaziz University (KAU) enrolled in the Faculties of Medicine, Dentistry, Pharmacology and Medical Technology.

\section{Methods}

\section{Study design \& participants}

This cross-sectional study was conducted among healthcare students at the largest University in Saudi Arabia, King Abdulaziz University (KAU). KAU has more than 40,000 students (2015/2016) enrolled in 18 faculties within three main areas: medicine, science, and humanities. All senior students enrolled in the medical stream including the Faculties of Medicine, Dentistry, Pharmacology and Medical Technology were targeted in this study. This study focused on senior students who were about to graduate at the end of the 2015/2016 academic year. These students were targeted due to their overall collective knowledge that has been accumulated during their medical studies and their upcoming involvement as future healthcare providers in Saudi Arabia.

\section{Questionnaire}

The data were collected using structured, self-administered questionnaires in the English language. The design of the questionnaire was based on surveys used in previously published studies $[8,9,26]$, and the questionnaire was enriched with additional questions to collect useful supplementary information. Questionnaires were randomly distributed and collected from students of each faculty. The investigator 
explained the purpose and significance of the study and informed students that participation in the study was voluntary and that all data will be anonymous and confidential. Ethical approval was obtained from the Research Ethics Committee at KAU Hospital (Ref. number: 106-15), and special permission to conduct the survey was obtained from each faculty. The three sections of the questionnaire were about (1) personal information and the general heath background of each participant, (2) biobanking knowledge, and (3) biomedical research attitudes.

\section{Participant biodata and general health status section}

This descriptive section collected data about participant socio-demographic characteristics and general health, including age, gender, faculty, school year, grade-point average (GPA; out of 5), marital status, number of children and general health status. In addition to inquiring about the health history of the students, including inherited diseases and/or hospitalization, this survey recorded their previous experience with blood or organ donation, genetic testing, and/or experience with participation in biomedical research.

\section{Biobanking knowledge questionnaire section}

This section consisted of two parts. The first included 7 main items that evaluated the general knowledge of the participants about biobanking and biospecimens and whether he/she knew about the following: HGP or biobanking terms, the definition of biobanking, the purpose of collecting biospecimens, the concept of consent, confidentiality, and the SOPs required for biospecimen collection.

The second part assessed the students' willingness to donate biospecimens to biobanks to perform biomedical research. It included questions about whether the participants were willing to donate tissue to biobanks, which specific tissue they were willing to donate (i.e., saliva, urine, blood, buccal swabs, toenails, hair, their own excess surgical tissue, or deceased family members' organs or tissues) and the reasons for their willingness or unwillingness to donate.

\section{Biomedical research attitudes questionnaire section}

This section focused on the Research Attitudes Questionnaire (RAQ), which is a validated questionnaire used to assess general attitudes towards biomedical research [26]. It is composed of 11 items listed on a 5-point Likert scale, with scores ranging from 1, "Strongly disagree", to 5, "Strongly agree". A total score was generated by summing all individual items, and higher scores indicate more positive attitudes.

\section{Statistical analysis}

The main outcomes (dependent) variables in this study were 1) knowledge about biobanking and 2) willingness to donate tissue to biobanks for biomedical research purposes. The willingness to donate was calculated as a binary variable (Yes/No) based on whether the respondent was willing to donate at least one of the specified tissues. For each of the 7 knowledge questions, a score of 1 was assigned if the participant gave a correct answer, and a score of 0 was assigned if the participant gave an incorrect answer. The percentage of participants who gave a correct answer for each knowledge question was calculated. In addition, for each participant, a total knowledge score was calculated by summing across questions, with scores ranging from a minimum of 0 to a maximum of 7 . The students' attitude towards biomedical research was measured using the RAQ. A total attitude score was generated based on the responses to each attitude item being rated on a 5-point Likert scale as follows: 1 "Strongly disagree", 2 "Disagree", 3 "Neutral", 4 "Agree", and 5 "Strongly agree". A total attitude score was calculated for each student by summing across items, with total scores ranging from a minimum of 5 to a maximum of 55 . Seven items were positively worded, and 4 were negatively worded. All negatively worded items were reversed such that a higher numbered response on the Likert scale would represent positive attitudes.

Categorical data were described using frequencies and percentages, whereas continuous data were described using means and standard deviations. The associations between predictors and willingness to donate were tested using the chi-squared test. The associations between knowledge and attitude scores and the willingness to donate were tested using the $t$-test. The significance level was set at 0.05. All statistical analysis was performed using STATA version 13 (StataCorp, College Station, Texas, USA).

\section{Results}

The questionnaire was completed by 597 of a cohort of 693 students, yielding a response rate of $86 \%$. The socio-demographic, health-related and biobankingrelated characteristics of the study population are summarized in Table 1. Females represented $61 \%$ of the cohort. Students attended the following faculties: Medicine (39\%), Dentistry (20\%), Pharmacology (13\%) and Medical Technology (28\%). The majority of respondents had a B grade average (53\%). Regarding their health status, 38\% reported excellent health status, whereas very few reported fair/poor health $(4 \%)$. Nine percent $(9 \%)$ of students reported being diagnosed with a chronic disease, $36 \%$ reported being hospitalized and $35 \%$ reported inherited diseases in their families. Approximately $91 \%$ have had a blood test and $28 \%$ have donated blood, whereas $16 \%$ have undergone a tissue test, and only $2 \%$ had already donated tissue. About half the students (45\%) have been involved in medical research. 
Table 1 Characteristics of the study population

Socio-demographic \&
Gender
Male
Female
Marital Status
Married
Non-married
Faculty
Medicine
Dentistry
Medical technology
Pharmacology
GPA
A
B
C/D

General Health

Excellent

Very good

Good

Fair/Poor

History of Chronic disease

$\begin{array}{ll}\text { No } & 528 \\ \text { Yes } & 51\end{array}$

Family history of inherited disease

$$
\begin{aligned}
& \text { No } \\
& \text { Yes }
\end{aligned}
$$

Previous hospitalization

$$
\text { No }
$$$$
\text { Yes }
$$

Biobanking-related variables

Previous genetic testing

$$
\text { No }
$$$$
\text { Yes }
$$

Previous blood testing

$$
\text { No }
$$

Yes

Previous tissue testing

$$
\begin{aligned}
& \text { No } \\
& \text { Yes }
\end{aligned}
$$

Previous blood donation
$N=597$
Table 1 Characteristics of the study population (Continued)

Previous tissue donation

\begin{tabular}{lll} 
No & 565 & 98 \\
Yes & 13 & 2 \\
nvolvement in medical research & & \\
No & 319 & 55 \\
Yes & 257 & 45 \\
\hline
\end{tabular}

Table 2 shows the students' responses to the biobanking knowledge questions. The mean knowledge score was $3.6 \pm 1.8$ out of 7 . Only $40 \%$ of students have heard of the HGP, whereas only $27 \%$ have heard of the term "biobanks". When asked about the purpose of biobanks, $59 \%$ correctly responded that it was to collect and store biospecimens for diagnosis, treatment and research purposes. Meanwhile, 30\% correctly defined biospecimens as being samples and/or biomolecules with annotated clinical, socioeconomic and lifestyle data. A high percentage of students (78\%) understood that donating 31 biospecimens to biobanks would require signing a con38 sent form, 57\% knew that their data would be kept confidential, and 65\% knew that there are SOPs for handling their donated biospecimens.

The majority of the students were willing to donate biospecimens to biobanks for biomedical research purposes (89\%). The associations between different variables and the willingness to donate biospecimens to biobanks are summarized in Table 3. Factors that

\begin{tabular}{|c|c|c|}
\hline Knowledge of Biobanking & $N$ & $\%$ \\
\hline Aware of the "Human Genome Project" & 257 & 44 \\
\hline Aware of the term "Biobank" & 157 & 27 \\
\hline $\begin{array}{l}\text { The purpose of biobank is to collect \& store } \\
\text { biospecimens for diagnosis, treatment and } \\
\text { research purposes }\end{array}$ & 339 & 59 \\
\hline $\begin{array}{l}\text { According to modern biobanking, biospecimens } \\
\text { are samples and/or biomolecules with annotated } \\
\text { clinical, socioeconomic and lifestyle data }\end{array}$ & 173 & 30 \\
\hline $\begin{array}{l}\text { Donating a biospecimen to a biobank requires } \\
\text { signing a consent form }\end{array}$ & 448 & 78 \\
\hline $\begin{array}{l}\text { There is a standard operating procedure (SOP) } \\
\text { for biobanks to collect, process, store and release } \\
\text { biospecimens }\end{array}$ & 373 & 65 \\
\hline $\begin{array}{l}\text { Biospecimen annotated data will be kept be } \\
\text { confidential and anonymous }\end{array}$ & 325 & 57 \\
\hline Biobanking knowledge score (mean \pm SD) & $3.2 \pm 1.6$ & \\
\hline
\end{tabular}
were significantly associated with the willingness to donate included marital status $(p$-value $=0.037)$, faculty $(p$-value $<0.001)$, general health status $(p$-value $=0.048)$, past experience with tissue testing and tissue donation $(p$-value $=0.042$ and $p$-value $<0.001$, respectively),

Table 2 Knowledge about biobanking 
Table 3 The associations between socio-demographic, health-related and biobanking-related variables and willingness to donate

\begin{tabular}{|c|c|c|c|c|c|}
\hline \multirow{3}{*}{ Variables } & \multicolumn{4}{|c|}{ Willingness to donate } & \multirow[t]{3}{*}{$p$-value } \\
\hline & \multicolumn{2}{|l|}{ Yes } & \multicolumn{2}{|l|}{ No } & \\
\hline & $\mathrm{N}$ & $\%$ & $\mathrm{~N}$ & $\%$ & \\
\hline Total & 517 & 89 & 66 & 11 & - \\
\hline \multicolumn{6}{|c|}{ Socio-demographic \& health-related } \\
\hline \multicolumn{6}{|l|}{ Gender } \\
\hline Male & 199 & 87 & 30 & 13 & 0.281 \\
\hline Female & 317 & 90 & 36 & 10 & \\
\hline \multicolumn{6}{|l|}{ Marital Status } \\
\hline Married & 57 & 81 & 13 & 19 & 0.037 \\
\hline Non-married & 459 & 90 & 52 & 10 & \\
\hline \multicolumn{6}{|l|}{ Faculty } \\
\hline Medicine & 193 & 85 & 35 & 15 & $<0.001$ \\
\hline Dentistry & 107 & 92 & 9 & 8 & \\
\hline Medical technology & 157 & 96 & 7 & 4 & \\
\hline Pharmacology & 60 & 80 & 15 & 20 & \\
\hline \multicolumn{6}{|l|}{ GPA } \\
\hline A & 167 & 91 & 16 & 9 & 0.394 \\
\hline $\mathrm{B}$ & 256 & 88 & 34 & 12 & \\
\hline$C / D$ & 67 & 86 & 11 & 14 & \\
\hline \multicolumn{6}{|l|}{ General Health } \\
\hline Excellent & 164 & 91 & 16 & 9 & $<0.001$ \\
\hline Very good & 200 & 91 & 20 & 9 & \\
\hline Good & 139 & 87 & 21 & 13 & \\
\hline Fair/Poor & 12 & 60 & 8 & 40 & \\
\hline \multicolumn{6}{|c|}{ History of Chronic disease } \\
\hline No & 470 & 89 & 58 & 11 & 0.554 \\
\hline Yes & 44 & 86 & 7 & 14 & \\
\hline \multicolumn{6}{|c|}{ Family history of inherited disease } \\
\hline No & 330 & 89 & 39 & 11 & 0.604 \\
\hline \multicolumn{6}{|l|}{ Yes } \\
\hline \multicolumn{6}{|l|}{ Previous hospitalization } \\
\hline No & 331 & 89 & 40 & 11 & 0.651 \\
\hline Yes & 183 & 88 & 25 & 12 & \\
\hline \multicolumn{6}{|l|}{ Biobanking-related } \\
\hline \multicolumn{6}{|l|}{ Previous genetic testing } \\
\hline No & 493 & 89 & 59 & 11 & 0.096 \\
\hline Yes & 23 & 79 & 6 & 21 & \\
\hline \multicolumn{6}{|l|}{ Previous blood testing } \\
\hline No & 45 & 88 & 6 & 12 & 0.887 \\
\hline Yes & 472 & 89 & 59 & 11 & \\
\hline \multicolumn{6}{|l|}{ Previous tissue testing } \\
\hline No & 437 & 90 & 49 & 10 & 0.042 \\
\hline Yes & 76 & 83 & 16 & 17 & \\
\hline
\end{tabular}


Table 3 The associations between socio-demographic, health-related and biobanking-related variables and willingness to donate (Continued)

\begin{tabular}{|c|c|c|c|c|c|}
\hline \multicolumn{6}{|l|}{ Previous blood donation } \\
\hline No & 370 & 89 & 45 & 11 & \multirow[t]{2}{*}{0.779} \\
\hline Yes & 144 & 88 & 19 & 12 & \\
\hline \multicolumn{6}{|l|}{ Previous tissue donation } \\
\hline No & 506 & 90 & 59 & 10 & \multirow[t]{2}{*}{$<0.001$} \\
\hline Yes & 8 & 62 & 5 & 39 & \\
\hline \multicolumn{6}{|l|}{ Involvement in medical research } \\
\hline No & 281 & 88 & 38 & 12 & \multirow[t]{2}{*}{0.495} \\
\hline Yes & 231 & 90 & 26 & 10 & \\
\hline Biobanking knowledge score (mean \pm SD) & \multicolumn{2}{|c|}{$3.17 \pm 1.6$} & \multicolumn{2}{|c|}{$2.33 \pm 1.6$} & $<0.001$ \\
\hline Biomedical research attitude score (mean \pm SD) & \multicolumn{2}{|c|}{$38.1 \pm 3.7$} & \multicolumn{2}{|c|}{$35.6 \pm 3.8$} & $<0.001$ \\
\hline
\end{tabular}

are considered statistically significant (Bold font)

knowledge of biobanking scores $(p$-value $<0.001)$ and biomedical research attitude scores ( $p$-value $<0.001)$. Factors such as gender, GPA, previous hospitalization, previous blood tests and blood donation were not significantly associated with the students' willingness to donate biospecimens.

Regarding the primary recorded reason for willingness/ unwillingness to donate biospecimens to biobanks, participants believed that donations would advance medical research and benefit society (44\%) (Table 4). Other reasons included the possibility of notification about abnormal results (25\%), benefiting them and their families (15\%), biobanks have already been established in developed countries

Table 4 Reasons for willingness or unwillingness to donate

\begin{tabular}{|c|c|c|}
\hline Reasons for willing to donate & $\mathrm{N}$ & $\%$ \\
\hline $\begin{array}{l}\text { The biobank will advance medical research, } \\
\text { benefit the society and future generation }\end{array}$ & 250 & 44 \\
\hline My family and I will benefit & 84 & 15 \\
\hline I could be notified about abnormal results & 143 & 25 \\
\hline $\begin{array}{l}\text { Samples will already be collected as part of } \\
\text { my medical care }\end{array}$ & 55 & 10 \\
\hline $\begin{array}{l}\text { Biobanks are already established as core facility } \\
\text { of biomedical research in developed countries }\end{array}$ & 68 & 12 \\
\hline Reasons for not willing to donate & $\mathrm{N}$ & $\%$ \\
\hline $\begin{array}{l}\text { Concern about misuse of biospecimen in } \\
\text { biomedical research }\end{array}$ & 88 & 15 \\
\hline $\begin{array}{l}\text { Concern about discovering genetic predispositions } \\
\text { to some diseases }\end{array}$ & 60 & 10 \\
\hline Concern about confidentiality & 71 & 12 \\
\hline $\begin{array}{l}\text { Concern that genetic information may be } \\
\text { used for discriminatory purposes }\end{array}$ & 41 & 7 \\
\hline $\begin{array}{l}\text { Concern that biospecimen may be used } \\
\text { for commercial purposes }\end{array}$ & 35 & 6 \\
\hline Fear of needles/injections & 76 & 13 \\
\hline Religious reasons & 16 & 3 \\
\hline
\end{tabular}

(12\%), and that samples will already be collected anyway for diagnostic purposes (10\%). The main reasons for unwillingness to donate were concerns about misuse of the biospecimens (15\%), followed by fear of needles/injections (13\%) and confidentiality concerns (12\%). Other reasons included fear of discovering genetic predispositions (10\%), concern that genetic information may be used for discrimination (7\%), concern that biospecimens may be used for commercial purposes (6\%) and religious reasons (3\%).

The tissues that students were willing to donate were mainly blood $(82 \%)$ and saliva/sputum (77\%). A considerable number of students were also willing to donate urine (70\%), buccal swabs (66\%), hair (67\%) and toenails (49\%). Despite the fact that the tissue was already designated for removal, only $43 \%$ of students were willing to donate their own excess tissue. A deceased family member's organs or tissues were the least desired for donation (25\%) (Fig. 1).

\section{Discussion}

PM primarily pertains to the identification of diagnostic strategies and the tailoring of therapeutic options customized to each individual according to their genetic background. However, the effectiveness of PM depends not only on the genomic signature of the patient but also on our understanding of the different interactions of genomic makeup with biology, lifestyle, wellbeing, and the overall environment. In this context, the emergence of biobanks as networks of biospecimen collections associated with relevant patient information is a cornerstone to the first decoding of human diseases at the molecular level and then refining our understanding of them using individual biodata. Elucidating this complex matching between biospecimens, patient information and OMICs-based data will ultimately help PM improve the current healthcare services offered to patients worldwide. Therefore, the education and awareness of healthcare providers are 


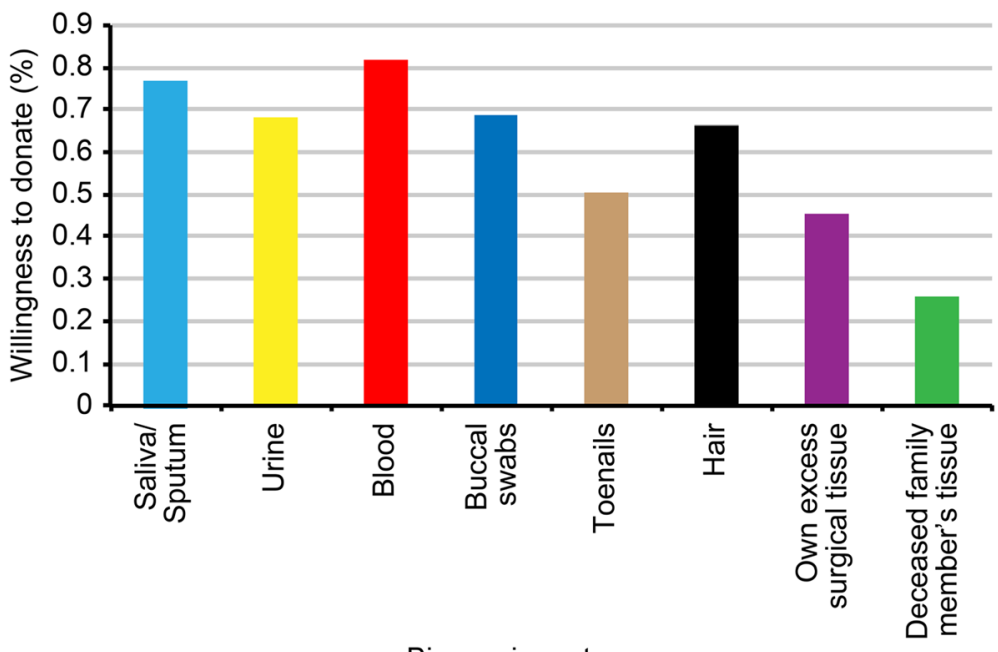

Biospecimen type

Fig. 1 Willingness to donate specific tissue

paramount to ensuring appropriate high-quality biospecimen management and real-time patient information collection according to the latest SOPs. Additionally, broad awareness campaigns among the public regarding the role and functions of biobanks can reinforce these worldwide efforts to deliver more efficient and precise care. An initial assessment of the current knowledge and attitudes about biobanks in a targeted geographical area is a prerequisite to tailoring suitable awareness programmes for the public or general healthcare providers. This type of assessment among senior healthcare students - who are future healthcare providers - is lacking in Saudi Arabia. Therefore, the objective of this questionnaire-based study was to assess the knowledge of senior healthcare students about biobanks, their willingness to donate biospecimens and the predictors of these attitudes to implement appropriate education and training programmes. The questionnaire was customized to collect students' biodata, health status, knowledge about biobanks and biospecimens, and willingness to donate biospecimens for biobanks and biomedical research.

The analysis indicated that students lacked sufficient knowledge about biobanks, as $73 \%$ of these future healthcare providers had never heard of the term "biobank". Moreover, $56 \%$ of the students had never heard of the HGP - the largest megaproject in biology in the last century - which has laid the foundation for several international collaborative and multidisciplinary initiatives to date [27].

Interestingly, and despite the noticeable lack of knowledge about the HGP and biobanks, our findings demonstrated that $89 \%$ of students were willing to donate biospecimens to biobanks. This higher enthusiasm of senior healthcare students at KAU to donate biospecimens is in agreement with previous findings from a local study conducted in Riyadh city (Saudi Arabia) and another survey conducted among the general public in Jordan, a neighbouring country with a similar culture [28]. Another study was conducted with patients, who were expected to be less aware of the HGP and biobanking; however, $70 \%$ of participants were willing to donate specimens [9]. Similar percentgaes of willingness to donate biospecimens (69\%) were reported in two different studies conducted in the USA, one with patients and the other with the Chinese American community $[14,29]$.

Several studies have investigated factors related to the willingness to donate to biobanks. Our data analysis indicated that such biospecimen donation willingness was significantly associated with several factors, mainly general health, marital status, faculty, past experience with tissue testing and/or tissue donation, biomedical attitude scores and knowledge of biobanking. In fact, we found that better self-reported health status was significantly associated with willingness to donate, and this finding is consistent with similar results reported from potential sample donors amongst the Swedish general population [7]. In addition, previous tissue tests and tissue donations were positive significant predictors in our study, with more than $62 \%$ of respondents being willing to donate their own future "extra" surgical tissue following the completion of all required medical diagnostics and therapeutics. However, in another local study targeting Saudi outpatients with a previous history of tissue tests and/ or donation, the percentage willing to donate biospecimens was only approximately $9 \%$ [9]. Compared with our results, this conspicuous difference might be because our cohort of interviewees consisted of senior healthcare students, who were therefore aware of the importance of voluntary tissue donations for the enhancing both biomedical research and clinical practice. 
To ensure suitable decision-making and customized awareness in healthcare students, an assessment of their knowledge and attitudes about biobanks and biomedical research was necessary [30]. Therefore, our questionnaire was designed to facilitate a better understanding of the factors influencing their attitudes and to transform their knowledge and willingness into measurable scores. In fact, the findings of the current study revealed that student knowledge of biobanking and biospecimens was insufficient, identifying a mean knowledge score of 3.6 out of 7. Approximately 59\% correctly defined the purpose of biobanks, and 30\% were able to guess the correct definition of biospecimens. This knowledge score was expected, as less than half of students had heard about the HGP, and approximately one third had heard of the term "biobank". In contrast, a study conducted among members of Kaiser Permanente in Colorado members who were approached in clinical waiting rooms found that the majority $(85 \%)$ of these patients - in contrast to our cohort composed of senior medical students - answered correctly when they were asked about general information regarding biospecimen collection for research purposes. This high percentage was attributed to the brochures and a draft consent form that were distributed to the respondents prior to answering the survey [14].

Measuring general attitudes towards biomedical research has also been found to significantly predict willingness to participate in biomedical research and donate biospecimens for research [26]. A Swedish study demonstrated that a positive public attitude towards genetic research was significantly associated with willingness to donate for research purposes [31]. An Italian survey reported that people who were willing to donate samples had a more positive attitude towards biomedical research than those who were not willing to donate [8]. Kobayashi et al. found that a positive attitude towards pharmacogenomics research was significantly associated with willingness to donate samples for biobanking [32]. Interestingly, the HGP outcomes and biobanking initiatives were shown to reinforce positive attitudes towards genetics and a greater willingness to donate biospecimens, as reflected in the Dutch public opinion in 2010 compared with that in 2002. The respondents exhibited higher expectations of biomedical research and thought that "genetic testing should be promoted more intensively" [33].

Regarding the factors related to willingness to donate biospecimens, our study demonstrated that participants' belief that "biobanks will advance medical research and benefit society and future generations" was the major factor influencing this willingness. The high biomedical research attitude score (38 out of 40) identified in this study was consistent with several local [34] and worldwide $[7,8,14,33,35]$ studies. A study conducted with Michigan college students found that many of them were very supportive of donating, hoping that it would benefit future patients [36]. The main reasons participants in this study were not willing to donate were concerns about misuse of biospecimens, worries about confidentiality and fear of needles/injections. Concerns about confidentiality have also been stated to be a main reason for refusing to donate in other studies elsewhere $[14,29]$ and were highlighted by other scientists and medical healthcare providers as evidence of the importance of building "trust" with the public [30,37-41]. In a conservative society, it was worth investigating the impact of religious beliefs, which were surprisingly reported to be the main reason for not willing to donate biospecimens by only $3 \%$ of the respondents. This contribution of religious beliefs in the decision to donate was lower than the percentage identified in another local study in 2009 targeting University students. This former study showed that approximately two-thirds of students linked their decisions to donate deceased relatives' tissues and organs with a jurisprudential opinion issued by religious experts/scholars [22]. This difference might be explained by the fact that the current study targeted senior students in the biomedical field. Additionally, the previous study was performed 7 years ago, and we think that since then, an important focus on genetics through social media has increased awareness of the importance of genetics and biomedical research among students attending KAU in 2016. However, this awareness remains too general and lacks the guidance and depth to grasp the importance of biobanking in the post-genomic era. A slight regional effect between Saudi Arabian regions might be involved, as suggested elsewhere [9]. Other studies in neighbouring countries have reported that $16 \%$ of medical students in Turkey were not willing to donate biospecimens for religious reasons, whereas $61 \%$ of the Jordanian public correlated their biospecimen donations with religious permission [23, 42].

In concordance with our findings, several studies have reported that scores for both biobanking knowledge and biomedical research attitudes were significantly higher in people willing to donate for research purposes than in those who were not. Goddard and colleagues in 2009 reported that participants who were willing to donate to biobanks were more likely to correctly answer knowledge questions [29], which supports our data from senior medical students. Level of education has also been significantly positively correlated with willingness to participate in biobanking specimen collection in Jordan [28].

This assessment of the current status of knowledge about biobanking and biomedical research sheds light on the necessity and opportunity to establish more personalized education and awareness strategies about PM and biobanking in the post-genomic era. An urgent revision of academic programmes delivered to medical students 
is needed to adopt the Double Helix Curriculum (DHC) by integrating of basic sciences and clinical biomedicine. Medical schools should also consider including teaching of advanced research methodology and OMICs-based technologies in their curricula [43]. More active involvement of students in biomedical research activities should be highly recommended and credited [30]. In parallel to this effort, additional awareness campaigns designed to raise public awareness about PM and biobanking and to highlight the importance of involvement are essential to building trustworthy partnerships [37, 44] for more effective biobank establishment and governance. This type of awareness programme is essential among healthcare providers and all biobanking stakeholders to bridge the gap between clinicians and scientists [45]. Therefore, deliberate policies and guidelines that will foster state-of-the art research in biomedical and clinical environments could be implemented to expedite the transition towards PM [46].

Several studies have reported significant effects of some of the predictors of willingness to donate biospecimens that were not significant in our survey. These factors include previous participation in medical research $[9,18]$, gender [29], age [7, 14, 16-19] and a history of previous hospitalization [9]. In addition to possible regional and cultural effects, these discrepancies between our results and the aforementioned previous reports targeting the general public (heterogeneous population) could be attributed to our population of interviewees, whio were exclusively senior health care students. In fact, the selection criteria stringency adopted in our questionnaire generated a sample of relatively homogenous individuals receiving similar curricula with comparable previous participation in medical research.

Regarding students' willingness to donate specific tissues, relatively high willingness rates were recorded for blood (82\%), saliva/sputum (77\%), urine (70\%), buccal swabs (66\%), hair (67\%) and toenails (49\%) (Figure 1). Willingness to donate was highest for blood donations, which was comparable to findings of other local studies [9]. This high percentage of blood donation willingness may be due to this population's familiarity with the concept of blood donation. In contrast, only about half the students in this study were willing to donate their own excess surgical tissue (43\%), which was lower than percentages in previous reports in which approximately $70 \%$ of both Swedish and Saudi respondents from the general public agreed to donate excess surgical tissue $[9,47]$. This finding was unexpected, especially from senior healthcare students who knew that these collected tissues would serve diagnostic and therapeutic purposes; only leftover tissue may be used for biomedical research following informed consent. This result could be due to the students' education level, which is associated with higher awareness of bioethics, confidentiality and possible misuse, as approximately $27 \%$ justified their attitude with concerns about confidentiality (12\%) or biospecimen misuse (15\%). These results highlighted an important issue related to the lack of biobanking knowledge and trust between stakeholders, including clinicians $[37,48]$. Therefore, creating future biobanking structures requires a comprehensive strategy that fosters trust between the public, healthcare providers, and policy makers to bridge the gap between scientists and clinicians and improve the welfare of the Saudi population. Other respondents reported concerns about discovering genetic predispositions to some diseases (10\%), and these concerns could be influenced by the relatively high rate of consanguinity in Saudi Arabia $[49,50]$.

Only a quarter of students were willing to donate a deceased family member's organs, and this percentage was comparable to that of other local studies targeting patients $[9,34]$. These local willingness percentages were lower than those reported worldwide. For example, a study on medical students in Pakistan reported that $45 \%$ were willing to donate their organs for transplantation [51]. Similar rates (48\%) were reported in the Swedish public, who stated that they would be prepared to allow a member of a research ethics committee to make decisions about the use of their own tissue when they weredeceased [47].

\section{Conclusion}

Since the publication of the first version of the human genome sequence, tremendous efforts have been made by the scientific community worldwide to uncover the genomic aetiology of diseases and to identify robust actionable and druggable targets for individualized therapeutics. These achievements have been attained thanks to the billions of biospecimens managed by biobanks in addition to the unprecedented outreach, education and networking amongst all stakeholders. The establishment of such an expanding international network was preceded by several comprehensive awareness programmes and workshops to foster the transition towards personalized healthcare [15]. Nevertheless, this transition revealed unexpected levels of genomic complexity that required additional international collaborative efforts along with broader awareness campaigns. The current study was designed to address this awareness gap in the MENA (Middle-East and North-Africa) region, specifically in Saudi Arabia, through a customized questionnaire targeting senior healthcare students. The results indicated a noticeable lack of knowledge about the HGP and biobanking amongst the respondents. However, the students expressed a high willingness to donate a wide range of biospecimens for biomedical research, and this willingness was significantly influenced by their knowledge of the HGP and biobanking scores, past experience with 
tissue test and/or tissue donation, biomedical attitude scores, and health status. Additional awareness and educational programmes tailored to these future healthcare providers to familiarize them with biobanking, biospecimen donation, genomics technologies and their scope in clinical applications are a paramount to helping students adhere to the PM international movement. Such awareness about the importance of informative involvement in biobanking, biospecimen donation and OMICs-driven medicine should also be expanded to all biobanking staff members, healthcare providers and the general public to foster state-of-the art biomedical research and to deliver clinically relevant applications that enhance the healthcare system.

\section{Abbreviations}

GPA: Grade-point average; HGP: Human genome project; KAU: King Abdulaziz University; PM: Precision medicine; RAQ: Research attitudes questionnaire; SOPs: Standard operating procedures

\section{Acknowledgements}

The authors also, acknowledge with thanks Science and Technology Unit, King Abdulaziz University for technical support.

\section{Funding}

This project was funded by the National Plan for Science, Technology and Innovation (MAARIFAH) - King Abdulaziz City for Science and Technology the Kingdom of Saudi Arabia - award number\# 11-BIO1512-03.

\section{Availability of data and materials}

The datasets during and/or analyzed during the current study available from the corresponding author on reasonable request.

\section{Authors' contributions}

LM contributed to the study design, data collection, data analysis and interpretation and manuscript writing; LA \& RG contributed to data collection and manuscript drafting; MA \& AB: Study design, data analysis and manuscript writing; SYS: contributed in data analysis and manuscript drafting; $A A \& M H Q$ : Obtaining funds, study supervision, technical and administrative support; JV: Data interpretation and critical revision of the manuscript. ALL authors critically reviewed and agreed on the final version of the manuscript.

\section{Competing interests}

The authors declare that they have no competing interests.

\section{Consent for publication}

Not applicable.

\section{Ethics approval and consent to participate}

The investigator explained the purpose and significance of the study and informed students that participation in the study was voluntary and that all data will be anonymous and confidential. Ethical approval was obtained from the Research Ethics Committee at KAU Hospital (Ref. number: 106-15), and special permission to conduct the survey was obtained from each faculty.

\section{Publisher's Note}

Springer Nature remains neutral with regard to jurisdictional claims in published maps and institutional affiliations.

\section{Author details}

${ }^{1}$ Department of Dental Public Health, Faculty of Dentistry, King Abdulaziz University, P.O. Box 80209, Jeddah 21589, Saudi Arabia. ${ }^{2}$ College of Public Health and Health Informatics, King Saud Bin Abdulaziz University for Health Sciences, P.O. Box 22490, Riyadh 11426, Saudi Arabia. ${ }^{3}$ Center of Excellence in Genomic Medicine Research, King Abdulaziz University, P.O. Box 80216, Jeddah 21589, Saudi Arabia. ${ }^{4}$ Center of Innovation in Personalized Medicine,
King Abdulaziz University, P.O. Box 80216, Jeddah 21589, Saudi Arabia. ${ }^{5}$ Department of Biology, Faculty of Science, King Abdulaziz University, Jeddah, Saudi Arabia. ${ }^{6}$ International Society for Biological and Environmental Repositories, Vancouver, BC V5Z 1B3, Canada. ${ }^{7}$ Biopreservation and Biobanking, Kensington, MD 20895, USA.

Received: 3 December 2016 Accepted: 27 April 2017

Published online: 02 May 2017

\section{References}

1. Gaskell G, Gottweis H, Starkbaum J, Gerber MM, Broerse J, Gottweis U, Hobbs A, Helen I, Paschou M, Snell K, et al. Publics and biobanks: panEuropean diversity and the challenge of responsible innovation. Eur J Hum Genet. 2013;21(1):14-20.

2. Wolf SM. Return of results in genomic biobank research: ethics matters. Genet Med. 2013;15(2):157-9.

3. Harris JR, Burton $P$, Knoppers BM, Lindpaintner K, Bledsoe $M$, Brookes AJ, Budin-Ljosne I, Chisholm R, Cox D, Deschenes M, et al. Toward a roadmap in global biobanking for health. Eur J Hum Genet. 2012;20(11):1105-11.

4. Christodoulou J. The human genome project: opportunities, challenges and consequences for population screening. Southeast Asian J Trop Med Public Health. 2003;34 Suppl 3:234-8.

5. Husedzinovic A, Ose D, Schickhardt C, Frohling S, Winkler EC. Stakeholders' perspectives on biobank-based genomic research: systematic review of the literature. Eur J Human Genet. 2015;23(12):1607-14.

6. Manolio TA, Abramowicz M, Al-Mulla F, Anderson W, Balling R, Berger AC, Bleyl S, Chakravarti A, Chantratita W, Chisholm RL, et al. Global implementation of genomic medicine: we are not alone. Sci Transl Med. 2015;7:290.

7. Dry SM, Garrett SB, Koenig BA, Brown AF, Burgess MM, Hult JR, Longstaff H, Wilcox ES, Madrigal Contreras SK, Martinez A, Boyd EA, Dohan D. Community recommendations on biobank governance: Results from a deliberative community engagement in California. PLoS One. 2017;24;12(2):e0172582.

8. Porteri C, Pasqualetti P, Togni E, Parker M. Public's attitudes on participation in a biobank for research: an Italian survey. BMC Med Ethics. 2014;15:81.

9. Al-Jumah M, Abolfotouh MA, Alabdulkareem IB, Balkhy HH, Al-Jeraisy MI, Al-Swaid AF, Al-Musaaed EM, Al-Knawy B. Public attitude towards biomedical research at outpatient clinics of King Abdulaziz Medical City, Riyadh, Saudi Arabia. East Mediterr Health J. 2011;17(6):536-45.

10. Mitchell D, Geissler J, Parry-Jones A, Keulen H, Schmitt DC, Vavassori R, Matharoo-Ball B. Biobanking from the patient perspective. Res Involv Engagem. 2015;1:1.

11. Cervo S, Rovina J, Talamini R, Perin T, Canzonieri V, De Paoli P, Steffan A. An effective multisource informed consent procedure for research and clinical practice: an observational study of patient understanding and awareness of their roles as research stakeholders in a cancer biobank. BMC Med Ethics. 2013;14:30.

12. Womack C, Mager SR, Womack C, Mager SR. Human biological sample biobanking to support tissue biomarkers in pharmaceutical research and development. Methods (San Diego, Calif). 2014:70(1):3-11.

13. Ragin C, Park JY. Biospecimens, biobanking and global cancer research collaborations. Ecancermedicalscience. 2014:8:454.

14. Rahm AK, Wrenn M, Carroll NM, Feigelson HS. Biobanking for research: a survey of patient population attitudes and understanding. J Community Genet. 2013:4(4):445-50.

15. Meir K, Gaffney EF, Simeon-Dubach D, Ravid R, Watson PH, Schacter B. Morente, the marble arch international working group on biobanking MM: the human face of biobank networks for translational research. Biopreservation Biobanking. 2011;9(3):279-85.

16. Gao W, Ma GX, Tan Y, Fang C, Weaver J, Jin M, Lai P. Factors associated with willingness to participate in biospecimen research among Chinese Americans. Biopreservation Biobanking. 2014;12(2):131-8.

17. McQuillan GM, Porter KS. Consent for future genetic research: the NHANES experience in 2007-2008. Irb. 2011;33(1):9-14.

18. Trauth JM, Musa D, Siminoff $L$, Jewell IK, Ricci E. Public attitudes regarding willingness to participate in medical research studies. J Health Soc Policy. 2000;12(2):23-43.

19. Malone T, Catalano PJ, O'Dwyer PJ, Giantonio B. High rate of consent to bank biologic samples for future research: the Eastern Cooperative Oncology Group experience. J Natl Cancer Inst. 2002;94(10):769-71.

20. Wang SS, Fridinger F, Sheedy KM, Khoury MJ. Public attitudes regarding the donation and storage of blood specimens for genetic research. Community Genet. 2001;4(1):18-26. 
21. Wong ML, Chia KS, Yam WM, Teodoro GR, Lau KW. Willingness to donate blood samples for genetic research: a survey from a community in Singapore. Clin Genet. 2004;65(1):45-51.

22. Al-Ghanim SA. The willingness toward deceased organ donation among university students. Implications for health education in Saudi Arabia. Saudi Med J. 2009;30(10):1340-5.

23. Sagiroglu M, Gunay O, Balci E. Attitudes of Turkish medical and law students towards the organ donation. Int J Organ Transplant Med. 2015;6(1):1-7.

24. Abu-Zaid A, Alkattan K. Integration of scientific research training into undergraduate medical education: a reminder call. Med Educ Online. 2013;18:22832

25. Solomon SS, Tom SC, Pichert J, Wasserman D, Powers AC. Impact of medical student research in the development of physician-scientists. J Investig Med. 2003;51(3):149-56.

26. Rubright JD, Cary MS, Karlawish JH, Kim SY. Measuring how people view biomedical research: Reliability and validity analysis of the Research Attitudes Questionnaire. J Empir Res Human Res Ethics. 2011;6(1):63-8.

27. Green ED, Watson JD, Collins FS. Human genome project: twenty-five years of big biology. Nature. 2015;526(7571):29-31.

28. Ahram M, Othman A, Shahrouri M. Public perception towards biobanking in Jordan. Biopreserv Biobank. 2012;10(4):361-5.

29. Goddard KA, Smith KS, Chen C, McMullen C, Johnson C. Biobank recruitment: motivations for nonparticipation. Biopreserv Biobank. 2009;7(2):119-21.

30. Feero WG, Green ED. Genomics education for health care professionals in the 21st century. JAMA. 2011;306(9):989-90.

31. Kettis-Lindblad $\AA$, Ring L, Viberth E, Hansson MG. Genetic research and donation of tissue samples to biobanks. What do potential sample donors in the Swedish general public think? Eur J Public Health. 2006;16(4):433-40.

32. Kobayashi $E$, Satoh N. Public involvement in pharmacogenomics research: a national survey on public attitudes towards pharmacogenomics research and the willingness to donate DNA samples to a DNA bank in Japan. Cell Tissue Bank. 2009;10(4):281-91.

33. Henneman L, Vermeulen E, van El CG, Claassen L, Timmermans DR, Cornel MC. Public attitudes towards genetic testing revisited: comparing opinions between 2002 and 2010. Eur J Human Genet. 2013;21(8):793-9.

34. Al-Jumah MA, Abolfotouh MA. Public perception and attitude of saudis toward organ and tissue donation. Biopreserv Biobank. 2011;9(1):21-7.

35. Simon CM, L'Heureux J, Murray JC, Winokur P, Weiner G, Newbury E, Shinkunas L, Zimmerman B. Active choice but not too active: public perspectives on biobank consent models. Genet Med. 2011;13(9):821-31.

36. Platt T, Platt J, Thiel DB, Fisher N, Kardia SL. 'Cool! and creepy': engaging with college student stakeholders in Michigan's biobank. J Commun Genet. 2014:5(4):349-62.

37. Moodley K, Singh S. "It's all about trust": reflections of researchers on the complexity and controversy surrounding biobanking in South Africa. BMC Med Ethics. 2016;17(1):57.

38. Vayena E, Gasser U. Between openness and privacy in genomics. PLoS Med. 2016;13:1.

39. Erlich Y, Williams JB, Glazer D, Yocum K, Farahany N, Olson M, Narayanan A, Stein LD, Witkowski JA, Kain RC. Redefining genomic privacy: trust and empowerment. PLoS Biol. 2014;12(11):e1001983.

40. Zarate OA, Brody JG, Brown P, Ramirez-Andreotta MD, Perovich L, Matz J. Balancing benefits and risks of immortal data: participants' views of open consent in the personal genome project. Hastings Cent Rep. 2016;46(1):36-45.

41. L'Heureux J, Murray JC, Newbury E, Shinkunas L, Simon CM. Public perspectives on biospecimen procurement: what biorepositories should consider. Biopreserv Biobank. 2013;11(3):137-43.

42. Ahram $M$, Othman A, Shahrouri M, Mustafa E. Factors influencing public participation in biobanking. Eur J Human Genet. 2014;22(4):445-51.

43. Green ED, Guyer MS. National Human Genome Research Institute: charting a course for genomic medicine from base pairs to bedside. Nature. 2011; 470(7333):204-13.

44. Critchley CR, Nicol D, Otlowski MF, Stranger MJ. Predicting intention to biobank: a national survey. Eur J Public Health. 2012;22(1):139-44.

45. Bjugn R, Casati B. Stakeholder analysis: a useful tool for biobank planning. Biopreserv Biobank. 2012;10(3):239-44.

46. Manolio TA, Green ED. Genomics reaches the clinic: from basic discoveries to clinical impact. Cell. 2011:147(1):14-6.

47. Hoeyer K, Olofsson BO, Mjorndal T, Lynoe N. Informed consent and biobanks: a population-based study of attitudes towards tissue donation for genetic research. Scand J Public Health. 2004;32(3):224-9.
48. Abu-Elmagd M, Assidi M, Dallol A, Buhmeida A, Pushparaj PN, Kalamegam G, Al-Hamzi E, Shay JW, Scherer SW, Agarwal A, et al. The third international genomic medicine conference (3rd IGMC, 2015): overall activities and outcome highlights. BMC Genom. 2016;17 Suppl 9:747.

49. Abu-Elmagd M, Assidi M, Schulten HJ, Dallol A, Pushparaj P, Ahmed F, Scherer SW, Al-Qahtani M. Individualized medicine enabled by genomics in Saudi Arabia. BMC Med Genom. 2015;8 Suppl 1:S3.

50. Turki RF, Assidi M, Banni HA, Zahed HA, Karim S, Schulten HJ, Abu-Elmagd $M$, Rouzi AA, Bajouh O, Jamal HS, et al. Associations of recurrent miscarriages with chromosomal abnormalities, thrombophilia allelic polymorphisms and/or consanguinity in Saudi Arabia. BMC Med Genet. 2016;17 Suppl 1:69.

51. Ali NF, Qureshi A, Jilani BN, Zehra N. Knowledge and ethical perception regarding organ donation among medical students. BMC Med Ethics. 2013;14:38.

\section{Submit your next manuscript to BioMed Central and we will help you at every step:}

- We accept pre-submission inquiries

- Our selector tool helps you to find the most relevant journal

- We provide round the clock customer support

- Convenient online submission

- Thorough peer review

- Inclusion in PubMed and all major indexing services

- Maximum visibility for your research

Submit your manuscript at www biomedcentral.com/submit
Ciomed Central 